\title{
Patterned stimuli in disinhibition and backward masking
}

\author{
DAVID BRYON \\ University of Houston at Clear Lake City, Houston, Texas 77058 \\ and \\ WILLIAM P. BANKS \\ Pomona College, Claremont, California 91711
}

\begin{abstract}
Different theories purporting to account for the disinhibition effect assume that masking and disinhibition are representative of the same class of phenomenon. This study provided one test of an alternative Gestalt-like hypothesis free of the assumption that disinhibition is a special case of masking. Although the results of this study did not support the hypothesis that Gestalt-like processes account for disinhibition, it was demonstrated that a disinhibition effect can be obtained using patterned stimuli for both targets and masks. A major implication of using patterned stimuli in disinhibition-type research is that it provides a methodology that minimizes or eliminates such extraneous cues as apparent movement, brightness reversals, and Mach bands.
\end{abstract}

The phenomenon of disinhibition is assumed to be a special case of backward masking in the visual system. When a second stimulus interferes with the perception of a first (target) stimulus, the second stimulus is called a masking stimulus, or simply a mask. If an additional stimulus is added to the two-stimulus sequence of target and mask, so that the first mask is followed by yet another visual stimulus (referred to as the second mask), the detection of the target stimulus may improve. This improvement in the detection of a target stimulus with the addition of a second mask is called disinhibition, or recovery.

The disinhibition phenomenon was discovered by Robinson (1966) in an experiment on backward masking for the purposes of determining what would happen to a target stimulus if a third stimulus was introduced at an interval short enough to interfere with the mask but not with the target. Robinson used three circular-shaped patches of light, varying in size, as the experimental stimuli. Two male subjects were instructed to identify each light patch with a numerical rating ( 1 for the smallest patch, 3 for the largest) and to report whether they saw 1,2 , or 3 , or any combination of light patches following each trial. Stimulus flashes were always of $20 \mathrm{msec}$ duration, as was the interval between the first mask and the second mask. Four different intervals $(25,50,75$, and $100 \mathrm{msec})$ were utilized between the target stimulus and the first mask. All viewing was monocular.

The combined data for both subjects clearly

Requests for reprints should be sent to William P. Banks, Department of Psychology, Pomona College, Claremont, California 91711. demonstrated the superiority of three-flash trials over two-flash trials in the identification of the target. In addition, when all three stimuli were presented in serial order, the first and third were reliably detected, but the second was not. Thus, according to Robinson (1966), by masking the second flash (first mask), the third flash (second mask) disinhibited, or recovered, the first flash (target stimulus).

Other researchers have confirmed and extended Robinson's (1966) findings by using different stimulus arrays for both target and masks as well as by varying stimulus duration times, interstimulus intervals (ISIs), and viewing conditions (Dember \& Purcell, 1967; Long \& Gribben, 1971; Mayzner, 1970; Purcell \& Dember, 1968; Purcell \& Stewart, 1975; Schiller \& Greenfield, 1969; Turvey, 1973). Theoretical accounts, however, of the assumed cognitive and physiological processes underlying masking and disinhibition remain tenuous and subject to wide dispute (Kahneman, 1968; Turvey, 1973).

It may be that masking and disinhibition are not representative of the same class of phenomenon. For example, with patterned targets and masks instead of amorphous stimuli like patches of light, disinhibition may result from a Gestalt-like process in which the two masks merge into a composite figure that is distinguishable from the target, with the time needed for the two masks to merge disrupting the effective temporal masking range of the target stimulus.

The purpose of this study was to provide one test of a theoretical Gestalt-like model for explaining disinhibition and to determine if the recovery phenomenon is restricted to experimental conditions that limit the number of patterned stimuli or if recovery would also be 
evidenced in instances in which all stimuli (targets and masks) were complex patterns varying in contour proximity.

\section{METHOD}

\section{Subjects}

Two males and one female served as subjects throughout the sequence of experiments. Subjects were paid and all came to the experimental setting untrained in tachistoscopic recognition tasks. All subjects had normal or corrected-to-normal visual acuity and were naive as to the purpose of the study.

\section{Apparatus}

Seven different patterned stimuli with varying contour properties were used in this study. The target stimuli were the letters $T$ and $L$, each $52 \mathrm{~min}$ of visual angle in height, $40 \mathrm{~min}$ in width, with a stroke of $2 \mathrm{~min}$. Mask 1 stimuli were two different patterned forms designated as "plain grid" and "diagonal grid" designs. Each Mask 1 pattern subtended 1.7 x 1.2 deg of visual angle and had a stroke width of 2 min. Mask 2 stimuli consisted of three different patterned forms designated as "overlapping letters," "random lines," and "solid figures" designs. All stimuli were high-contrast pen and black ink drawings on white pieces of cardstock.

All stimuli were presented binocularly in a four-field Iconix tachistoscope with associated timers and power supplies. Stimuli were centered in $10 \times 10 \mathrm{deg}$ visual fields, in which subjects viewed them from a distance of $86 \mathrm{~cm}$ at a luminance of $15 \mathrm{~mL}$ $\left(55.3 \mathrm{~cd} / \mathrm{m}^{2}\right)$.

\section{Procedure}

In the four experiments, subjects were allowed a 5 -min adaptation period in the experimental setting. Following the adaptation period, subjects were given a 25 -trial preliminary practice session that allowed for any seating adjustments and ensured that subjects had understood all instructions. In all four experiments, the number of trials for each experimental condition was fixed at 20. In Experiments 1 and 2, a dim red fixation point from a single red light-emitting diode (dominant wavelength of $630 \mathrm{~nm}$ ) defined the center of the target area. In Experiments 3 and 4 , subjects oriented to the visual field without the presence of a fixation point.

A forced-response procedure was employed in all experiments. Subjects reported the stimuli verbally and were instructed to make a best guess if any of the stimuli were unclear. Each stimulus presentation was preceded by the experimenter saying "ready." In Experiments 3 and 4, subjects were also instructed to fixate in the center of the viewing field, in which all stimuli occurred in the same spatial location.

Experiment 1. The purpose of this experiment was to determine the masking efficacy of the two Mask 1 patterned stimuli on the alphabetic target stimuli and to articulate ISIs and stimulus durations for maximal masking efficiency.

The subjects' task was to make forced-choice identifications of the target letters $\mathrm{T}$ or $\mathrm{L}$ following the masking sequence. Target and Mask 1 stimuli were presented according to a random sequence in conjunction with a range of ISIs $(1,5,10,20,30$, 80 , or $120 \mathrm{msec}$ ) and stimulus durations $(10$ or $20 \mathrm{msec})$. Parameters for target and mask presentations were determined which yielded approximately $75 \%$ forced-choice target identification accuracies at specified ISI and stimulus duration values. Both Mask 1 patterned stimuli displayed effective masking characteristics.

Experiment 2. The purpose of this experiment was to determine the masking efficacy of the three Mask 2 patterned stimuli on the two Mask 1 stimuli and to articulate ISIs and stimulus durations for maximal masking efficiency.

The subjects' task was to make a forced-choice identification of the target stimuli by responding "one" to the plain grid stimulus and "two" to the diagonal grid stimulus following the masking sequence. Target and masking stimuli were randomly presented in conjunction with a range of ISIs $(1,5,10,20,40$, 80 , and $120 \mathrm{msec}$ ) and fixed stimulus durations of $20 \mathrm{msec}$. Parameters for target and mask presentations were determined which yielded approximately $75 \%$ forced-choice target identification accuracies at specific ISI and 20-msec stimulus duration values. All three Mask 2 stimuli showed effective masking abilities.

Experiment 3. The purpose of this experiment was to determine if the recovery phenomenon would be evidenced by a three-stimulus presentation of target and masks, and, if so, what general range of ISIs and stimulus durations would be most effective.

The subjects' task was to make forced-choice identifications of the target stimuli ( $\mathrm{T}$ or $\mathrm{L}$ ) following three-stimulus presentations. Both mask durations were set at $20 \mathrm{msec}$ with a fixed ISI of $40 \mathrm{msec}$ between them. Target durations and ISI values between target and Mask 1 were varied in tandem at 10,20,30, and $40 \mathrm{msec}$ and presented randomly. A control condition of target plus Mask 1 was randomly presented with the same target duration and ISI values being varied as in the three-stimulus condition. Random trials of target $(10 \mathrm{sec})$ plus Mask $2(20 \mathrm{sec})$, with an ISI of $70 \mathrm{msec}$ between them, were also presented to assess any possible direct effects of Mask 2 on the target stimulus.

The overall mean number of correct target identifications for the three-stimulus (disinhibition) sequence in comparison with the overall mean number for the control (target plus Mask 1) condition indicated that at the $10-\mathrm{msec}$ value for target duration and first ISI, a higher rate of correct target identifications occurred in the disinhibition sequence than in the control condition. Because target durations and ISIs for Mask 1 were varied in tandem, it remained to be determined which factor (target duration or ISI) might best account for the recovery effect. No direct effects of Mask 2 on the target stimuli were evidenced in the target plus Mask 2 condition.

Experiment 4. The purpose of this experiment was to confirm the recovery phenomenon for patterned stimuli and to determine the relative contributions of target durations and ISIs to the effect.

The subjects' task was to make forced-choice identifications of the target stimuli ( $T$ or $L$ ) following three-stimulus presentations. The procedures followed were exactly the same as those in Experiment 3. The target plus Mask 2 sequence was not repeated. Target duration for the recovery sequence was fixed at $10 \mathrm{msec}$, and ISIs between target and Mask 1 were varied at $10,20,30$, and $40 \mathrm{msec}$. Mask durations $(20 \mathrm{msec})$ and their ISI $(40 \mathrm{msec})$ were the same as in Experiment 3 . The control condition (target plus Mask 1) varied over an identical range of target and Mask 1 durations and ISIs as those in the threestimulus condition.

\section{RESULTS}

The accuracy rate, defined as the percent of correct target identifications (50\% indicates chance), was determined for each subject over all factors (type of Mask 1, type of Mask 2, and ISI values) and entered into analysis of variance. Significant main effects were found among Mask 1 conditions $[F(1,2)=27.92$, $\mathrm{p}<.05]$, Mask 2 conditions $[\mathrm{F}(3,6)=8.00, \mathrm{p}<.05]$, and ISIs $[F(3,6)=110.24, p<.01]$. A significant interaction was evidenced between Mask 2 conditions and ISIs as well $[\mathrm{F}(9,18)=4.73, \mathrm{p}<.01]$.

Since the comparison of the experimental conditions 


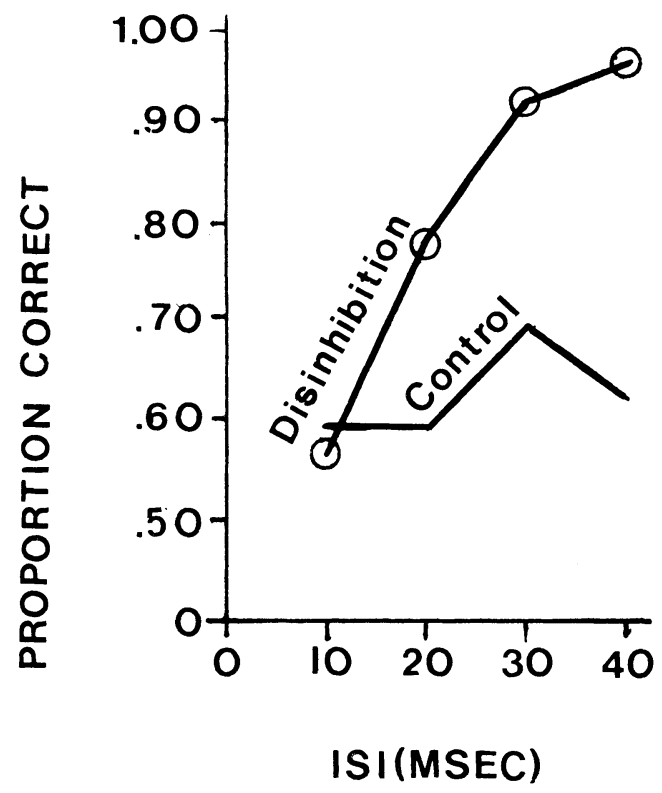

Figure 1. Target identification accuracies.

(three-stimulus presentations) with the control condition (target plus Mask 1) was of most interest to this study, simple main effects were determined by comparing the control condition with the average of the experimental conditions at each ISI. At the ISI level of $10 \mathrm{msec}$, the $\mathrm{t}$ test comparing the control condition with the average accuracy rate of the three recovery conditions was not significant $[\mathrm{t}(24)=.39]$. However, $t$ tests comparing the control condition with the averaged accuracy rates for the recovery conditions confirmed disinhibition effects at the $20-\mathrm{msec}$ $[\mathrm{t}(24)=-3.26, \mathrm{p}<.01], \quad 30-\mathrm{msec} \quad[\mathrm{t}(24)=-4.04$, $\mathrm{p}<.001]$, and 40-msec $[\mathrm{t}(24)=-5.84, \mathrm{p}<.001]$ ISI values. A further test showed that the target plus Mask 1 condition was not affected by varying levels of ISI $[F(3,24)=1.09$, n.s. $]$. Thus, whereas the average of the experimental conditions showed a highly significant effect of ISI, the differences among conditions arose primarily from differences between the control condition and the recovery condition (see Figure 1).

\section{DISCUSSION}

In this study, three configurationally different types of patterned stimuli (overlapping letters, random lines, and solid figures), which were comparable in their ability to mask other patterned stimuli (plain grid or grid with diagonals), proved to be similarly effective in their ability to recover a target (the letter $\mathrm{T}$ or $\mathrm{L}$ ) in a disinhibition-type sequence. Thus, a recovery effect has now been demonstrated that utilizes patterned stimuli for both masks in the disinhibition sequence, not just Mask 1 (Mayzner, 1970; Purcell \& Stewart, 1975; Turvey, 1973).

One of the major implications of two-patterned masking for disinhibition-type research is that it introduces a methodology for studying disinhibition that minimizes or completely eliminates certain extraneous cues, such as apparent movement, luminance summation, Mach bands, and brightness reversals, which may have confounded the findings of such researchers as Robinson (1966, 1968) and Schurman and Eriksen (1969).

Theoretical positions seeking to explain masking and disinhibition phenomena may be grouped into three categories (Kahneman, 1969; Weisstein, 1968): integration, interruption, and lateral inhibition. Integration theory assumes that masking results from the mask combining with the target such that the target is no longer perceptually distinguishable from the mask that followed it. Interruption theory posits that either the neural representation of the target is erased by the mask or the mask disrupts the information contained in the target. Lateral inhibition theory is based on the known interaction of neural receptive fields in the visual system and assumes that masking effects result from the inhibitory influences of one receptive field upon another. Thus, in seeking to explain the recovery effect, one may postulate that Mask 2 releases Mask 1's suppression of the target (lateral inhibition), that Mask 2 prevents Mask 1 from distracting a central processor while analyzing the target (integration), or that Mask 2 catches up with Mask 1, erases it, and restores the target (interruption).

These theoretical accounts of disinhibition have rested on the assumption that masking and recovery are representative of the same class of phenomenon. Another model, however, suggests itself, one that posits a Gestalt-like process as underlying the recovery effect and does not assume disinhibition to be a special case of masking. In a Gestalt-like model of disinhibition, Masks 1 and 2 would merge into a composite figure distinguishable from the target, and the temporal processing requisite for the merging of the masks would disrupt the effective masking range of the Mask 1 stimulus on the target stimulus.

One test of this Gestalt hypothesis would be to equate different patterned stimuli in terms of their masking abilities but to vary stimulus contours, which would have the effect of varying the likelihood with which the different stimuli formed whole figure composites. In the present study, different types of patterned stimuli, all of which varied in contour proximity, were equated for their ability to mask. If masking and disinhibition represent different classes of phenomena, then patterned stimuli with equivalent masking abilities should have equivalent disinhibiting abilities. If masking and disinhibition represent different classes of phenomena and a Gestalt-like process holds, then differential recovery effects should be evidenced with different types of patterned stimuli, even though their masking abilities are equivalent.

The results of this study indicated that patterned stimuli with equivalent masking abilities yielded equivalent recovery effects. This finding does not support the hypothesis that a Gestalt-like process accounts for recovery with patterned stimuli. It does, however, support those theoretical positions, such as lateral inhibition theory, which assert that masking and disinhibition represent the same class of phenomenon. On the other hand, the success of complex patterned stimuli with dissimilar contour proximities argues against simple brightness reversals at the retinal level as the basis for recovery (Barry \& Dick, 1972; Purcell \& Dember, 1968) and suggests, along with Turvey (1973), that some sort of central processing might be necessary to account for disinhibition with patterned stimuli under dichoptic viewing conditions.

\section{REFERENCES}

BARry, S. H., \& Dick, A. O. On the "recovery" of masked targets. Perception \& Psychophysics, 1972, 12, 117-120.

Dember, W. N., \& Purcell, D. G. Recovery of masked visual targets by inhibition of the masking stimulus. Science, 1967, 157, 1335-1336.

KaHneman, D. Method, findings, and theory in studies of visual masking. Psychological Bulletin, 1968, 70, 404-425.

Long, N. R., \& Gribien, J. A. The recovery of a visually 
masked target. Perception \& Psychophysics, 1971, 10, 197-200. MAYZnER, M. S. The disinhibited effect in sequential blanking. Psychonomic Science, 1970, 20, 218-219.

Purcell, D. G., \& Dember, W. N. The relation of phenomenal brightness reversal and re-reversal to backward masking and recovery. Perception \& Psychophysics, 1968, 3, 290-292.

Purcell, D. G., \& Stewart, A. L. Visual masking by a patterned stimulus and recovery of observer performance. Bulletin of the Psychonomic Society, 1975, 6, 457-460.

Robinson, D. N. Disinhibition of visually masked stimuli. Science, 1966, 154, 157-158.

Robinson, D. N. Visual disinhibition with binocular and interocular presentations. Journal of the Optical Society of America, $1968,58,254-257$.
Schiller, P. H., \& Greenfield, A. Visual masking and the recovery phenomenon. Perception \& Psychophysics, 1969, 6, 182-184.

Schurman, D. L., \& Eriksen, C. W. Summation and interaction of successive masking stimuli in visual perception. American Journal of Psychology, 1969, 82, 320-332.

Turvey, M. T. Of peripheral and central processes in vision. Psychological Review, 1973, 80, 1-52.

Weisstein, N. A Rashevsky-Landahl neural net: Simulation of metacontrast. Psychological Review, 1968, 75, 494-521.

(Received for publication November 21, 1979.) 